\title{
Hypoxia-inducible factor-1a-dependent induction of miR122 enhances hepatic ischemia tolerance
}

\author{
Cynthia Ju, ${ }^{1}$ Meng Wang, ${ }^{1}$ Eunyoung Tak, ${ }^{2}$ Boyun Kim, ${ }^{1}$ Christoph Emontzpohl, ${ }^{1}$ Yang Yang, ${ }^{1}$ Xiaoyi Yuan, ${ }^{1}$ Huban Kutay, ${ }^{3}$ \\ Yafen Liang, ${ }^{1}$ David R. Hall, ${ }^{4}$ Wasim A. Dar, ${ }^{4}$. Steve Bynon, ${ }^{4}$ Peter Carmeliet, ${ }^{5,6}$ Kalpana Choshal, ${ }^{3,7}$ and Holger K. Eltzschig' \\ 'Department of Anesthesiology, McCovern Medical School, University of Texas Health Science Center at Houston, Texas, USA. ${ }^{2}$ Asan Institute for Life Sciences, Asan Medical Center, University of Ulsan College \\ of Medicine, Seoul, South Korea. ${ }^{3}$ Comprehensive Cancer Center, The Ohio State University, Columbus, Ohio, USA. ${ }^{4}$ Department of Surgery, McCovern Medical School, University of Texas Health Science Center \\ at Houston, Houston, Texas, USA. ${ }^{5}$ Laboratory of Angiogenesis and Vascular Metabolism, Department of Oncology, and ${ }^{6}$ Center for Cancer Biology, Department of Oncology, Katholieke University Leuven, \\ Leuven, Belgium. Department of Pathology, The Ohio State University, Columbus, Ohio, USA
}

\begin{abstract}
Hepatic ischemia and reperfusion (IR) injury contributes to the morbidity and mortality associated with liver transplantation. microRNAs (miRNAs) constitute a family of noncoding RNAs that regulate gene expression at the posttranslational level through the repression of specific target genes. Here, we hypothesized that miRNAs could be targeted to enhance hepatic ischemia tolerance. A miRNA screen in a murine model of hepatic IR injury pointed us toward the liver-specific miRNA miR122. Subsequent studies in mice with hepatocyte-specific deletion of miR122 (miR122 ${ }^{\text {loxP/loxP }} A / b-C r e^{+}$mice) during hepatic ischemia and reperfusion revealed exacerbated liver injury. Transcriptional studies implicated hypoxia-inducible factor-1 $\alpha$ (HIF1 $\alpha$ ) in the induction of miR122 and identified the oxygen-sensing prolyl hydroxylase domain 1 (PHD1) as a miR122 target. Further studies indicated that HIF1 $\alpha$-dependent induction of miR122 participated in a feed-forward pathway for liver protection via the enhancement of hepatic HIF responses through PHD1 repression. Moreover, pharmacologic studies utilizing nanoparticle-mediated miR122 overexpression demonstrated attenuated liver injury. Finally, proof-of-principle studies in patients undergoing orthotopic liver transplantation showed elevated miR122 levels in conjunction with the repression of PHD1 in post-ischemic liver biopsies. Taken together, the present findings provide molecular insight into the functional role of miR122 in enhancing hepatic ischemia tolerance and suggest the potential utility of pharmacologic interventions targeting miR122 to dampen hepatic injury during liver transplantation.
\end{abstract}

\section{Introduction}

Hepatic ischemia and reperfusion (IR) injury occurs during hemorrhagic shock, trauma, liver resection, and liver transplantation surgery $(1,2)$. Early graft failure after liver transplantation is frequently caused by IR injury and is associated with extremely high rates of morbidity and mortality (3). Because of the growing waitlist of recipients whose need for liver transplantation is urgent, there is an increasing demand for liver grafts for transplantation. Although they are more prone to IR injury, marginal livers are now also more frequently being considered for transplantations (4). Hepatic IR injury also has important immunologic consequences, such as an increased severity of early liver rejection (5) or the subsequent recurrence of viral hepatitis (6). Therefore, the search for novel therapeutic interventions to dampen hepatic IR injury is an area of intense investigation (1, 7-14).

MicroRNAs (miRNAs) constitute a family of short, noncoding RNA molecules of 20 to 25 nucleotides in length that regulate gene expression at the posttranscriptional level via repression of target

Authorship note: CJ, MW, and ET are co-first authors.

Conflict of interest: The authors have declared that no conflict of interest exists. Copyright: (5) 2021, American Society for Clinical Investigation.

Submitted: May 18, 2020; Accepted: February 10, 2021; Published: April 1, 2021.

Reference information: J Clin Invest. 2021;131(7):e140300.

https://doi.org/10.1172/JCl140300. genes. miRNAs are involved in the control of a wide range of biological functions and processes, such as development, differentiation, metabolism, growth, proliferation, and apoptosis (15). The contributions of miRNAs to hepatic IR injury are largely unknown. In order to identify miRNAs that could be targeted for liver protection during IR injury, we performed a targeted miRNA array using a murine model of hepatic IR injury. Interestingly, these studies pointed us toward miR122, a highly conserved liver-specific miRNA that constitutes $70 \%$ of the cloned hepatic miRNA in the adult mouse (16). Several key observations underscore the importance of miR122 in liver biology and disease. Antisense-mediated inhibition of miR122 in mice leads to the induction of genes that are normally repressed in adult liver, suggesting that this miRNA is important for the maintenance of the terminally differentiated hepatocyte gene expression program (17). Furthermore, miR122 inhibition reduces serum cholesterol by indirectly causing the repression of genes involved in cholesterol biosynthesis, thereby protecting animals from diet-induced hypercholesterolemia (18). Additionally, miR122 plays a noncanonical role in the life cycle of hepatitis $\mathrm{C}$ virus (HCV) and its replication (19). Accordingly, intravenous administration of locked nucleic acid (LNA) antisense miR122 oligonucleotides (miravirsen) was found to be therapeutic in the treatment of patients with hepatitis C (20). A recent study showed that injection of pre-miR122-expressing adeno-associated virus serotype 8 (AAV8) to mice ameliorated alcoholic liver disease, suggesting a protective function of miR122 (21). 

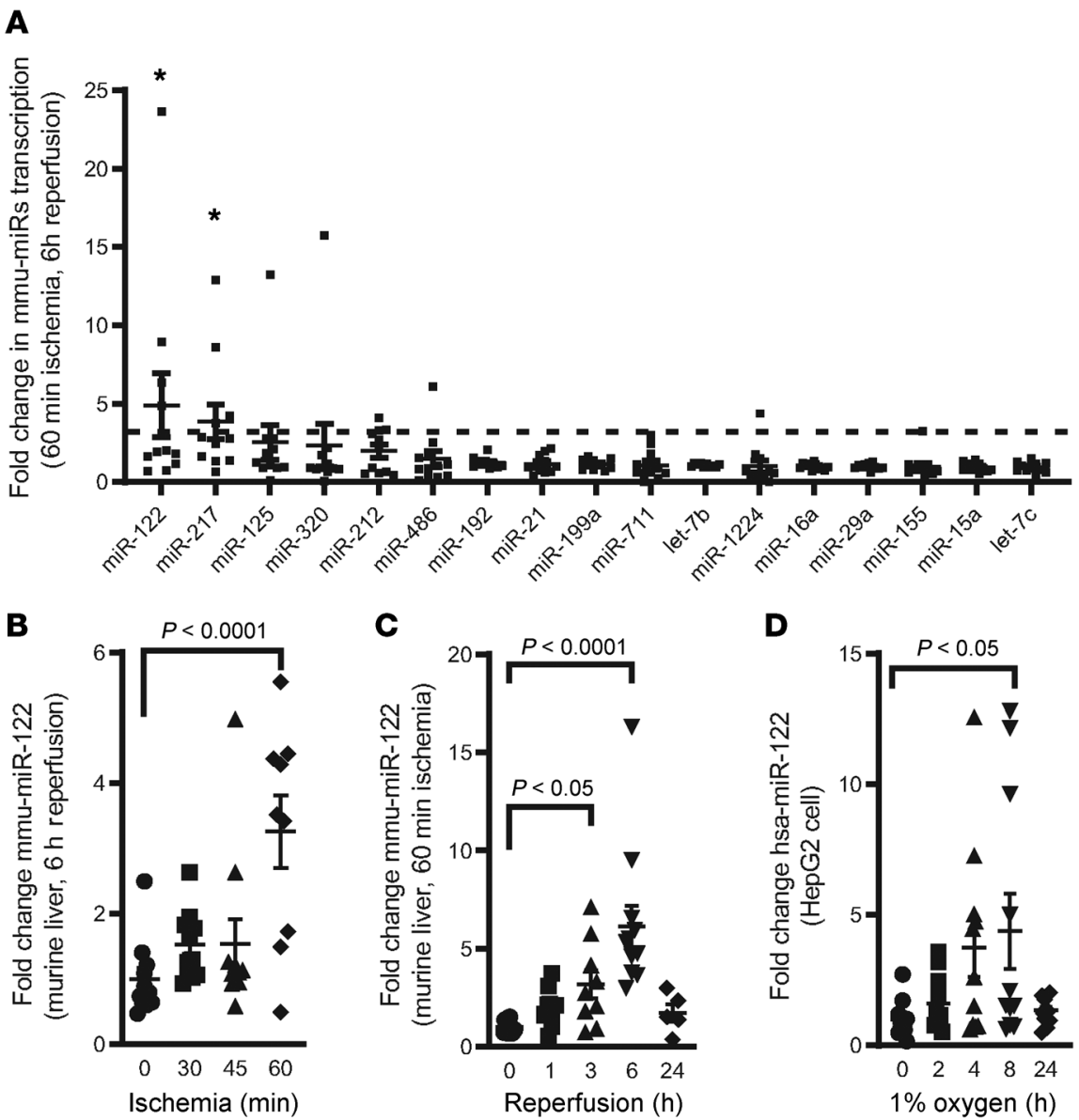

Figure 1. Identification of miR122 as a hypoxiaresponsive miRNA during hepatic IR injury.

(A) Targeted miRNA array in WT liver after hepatic ischemia (60 minutes) and reperfusion (6 hours). The data shown indicate fold changes of the indicated miRNAs in mouse livers subjected to IR injury relative to sham control liver tissues. ${ }^{*} P<$ 0.05 compared with values for the WT sham mice indicated by the dashed line, by 2-tailed, unpaired Student's $t$ test. $n=11 /$ group. (B) Hepatic mmumiR122 transcript levels following the indicated hepatic ischemia times and a 6-hour reperfusion. Statistical significance was determined by 1-way ANOVA. $n=9 /$ group, except $n=10$ in the 30-minute ischemia group. (C) Hepatic mmu-miR122 transcript levels after 60 minutes of liver ischemia and the indicated reperfusion times. Statistical significance was determined by 1-way ANOVA. $n=$ $13,9,9,12$, and 5 for $0,1,3,6$, and 24 hours, respectively. (D) Hsa-miR122 transcript levels in cultured human hepatocytes (HepG2 cells) exposed to hypoxia ( $1 \%$ oxygen) for the indicated durations. Statistical significance was determined by 1-way ANOVA. $n=12,12,11,11$, and 11 for the $0-, 2-, 4-$, 8-, and 24-hour groups, respectively). All data are shown as the mean \pm SEM.
miR122 represents a specific biomarker for acute and chronic liver injury, owing to its almost exclusive expression in hepatocytes. Elevated serum levels of miR122 have been reported in drug-induced liver injury $(22,23)$, alcoholic liver disease $(24)$, HCV (25, 26), and nonalcoholic steatohepatitis (NASH) (27). Increase levels of miR122 in the serum have also been observed in mouse and rat models of warm hepatic IR injury $(28,29)$. The data demonstrated a strong correlation between serum levels of miR122 and the degree of liver damage measured by alanine aminotransferase (ALT), aspartate aminotransferase (AST), and hepatic cell death. A recent clinical study of liver grafts at the end of cold ischemia demonstrated that the miR122 levels in preservation fluids were significantly higher in grafts from donation after circulatory death (DCD) and grafts that developed early allograft dysfunction (EAD). Moreover, the levels of miR122 in preservation fluids correlated with ALT levels within the first day after transplantation and reduced long-term graft survival (30). These studies suggest that circulating miR122 levels could be a potential biomarker of hepatic IR injury. However, the function of miR122 in hepatic IR injury and its potential as a therapeutic target have not been elucidated. The present studies demonstrate a protective role for miR122 in promoting hepatic ischemia tolerance.

\section{Results}

MiR122 is upregulated during hepatic IR injury. We hypothesized that miRNAs could play a critical role in mediating hepatic ischemia tolerance via alteration of the expression of specific tar- get genes. To examine this hypothesis, we performed a targeted miRNA array using mouse liver tissue after hepatic IR injury, focusing on miRNAs that are known to be expressed in the liver (31-34). For the purpose of these studies, we exposed mice to partial hepatic IR injury by applying 60 minutes of hepatic ischemia of the left and middle liver lobes, followed by 6 hours of reperfusion. We compared miRNA expression in the post-IR liver with expression in sham control livers. Interestingly, we observed the most robust induction of miRNA expression for the hepatic miRNA mmu-miR122 (Figure 1A). Additional studies with different ischemia or reperfusion durations confirmed increased mmu-miR122 expression following IR injury of the liver (Figure 1, B and C). Moreover, exposure of the human hepatocyte cell line HepG2 to ambient hypoxia (1\% oxygen) was associated with time-dependent increases in hsa-miR122 expression (Figure 1D). Together, these findings demonstrated miR122 induction during hepatic IR injury.

Upregulation of miR122 during hepatic IR injury is mediated by hypoxia-inducible factor-1 $\alpha$. On the basis of the above findings showing that miR122 was induced during murine hepatic IR injury, or following hypoxia exposure of HepG2 cells, we next set out to study the transcriptional mechanism governing miR122 expression. Unlike some miRNAs, which are encoded on transcripts coincident with other miRNAs or protein-coding genes, miR122 is derived from a transcript that only encodes for miR122. Previous studies have shown that the miR122 promoter is prom- 
A

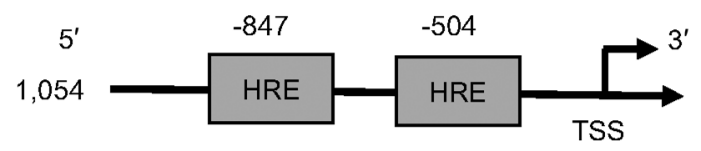

B

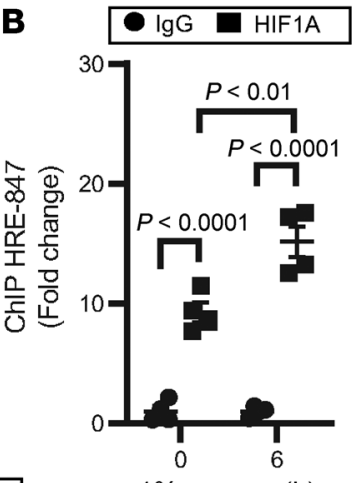

$1 \%$ oxygen (h)

E

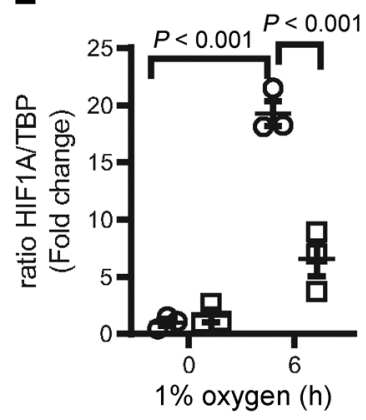

I

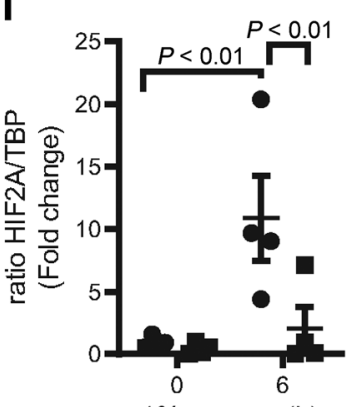

$1 \%$ oxygen (h)

M

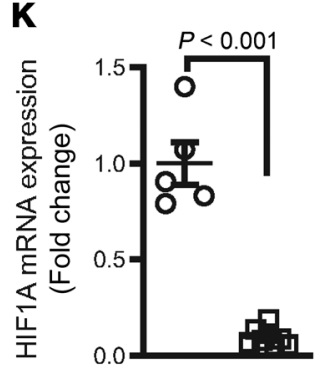

$\mathbf{L}$

H

HIF2A

TBP

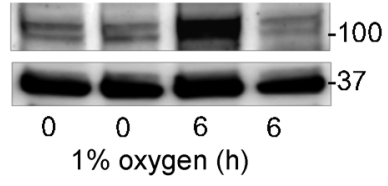

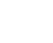

$$
\frac{\text { Alb-Cre }}{\text { sham IR }} \frac{{\text { HIF } 1 A^{\text {tllf }} \text { Alb-Cre }}^{+}}{\text {sham IR }}
$$

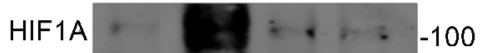

TBP

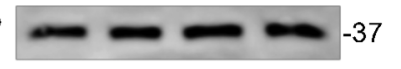

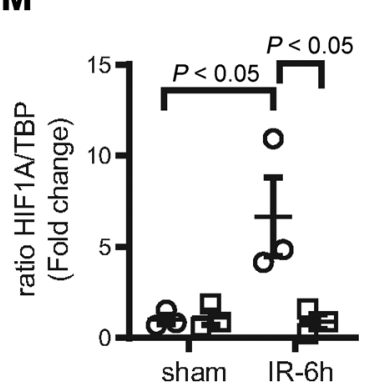

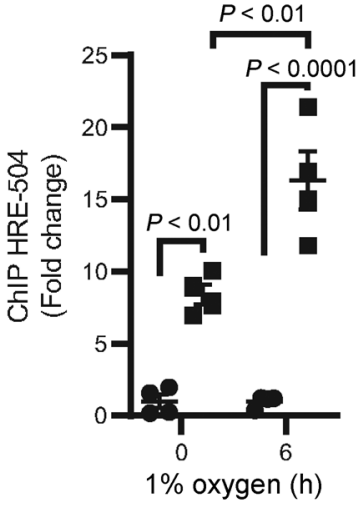

$\mathbf{F}$

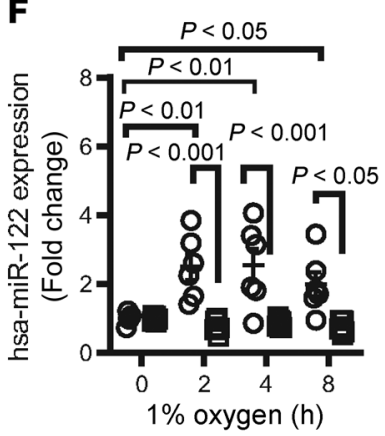

$\mathbf{J}$

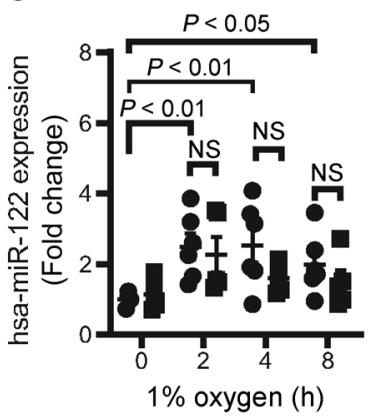

$\mathbf{N}$

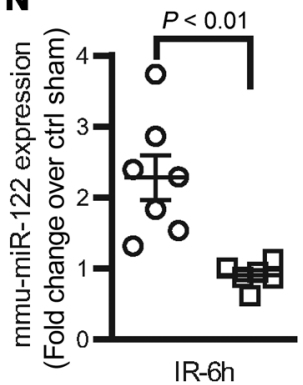

Figure 2. Role of HIF in miR122 induction. (A) The promoter for miR122 includes 2 putative HIF-binding sites - hypoxia response elements (HREs). Maps indicate the promoter constructs generated for analysis of promoter activity. (B) ChIP analysis demonstrated binding of HIF1 $\alpha$ to the putative HRE within the miR122 promoter (2-way ANOVA. $n=4$ /group from 4independent experiments). (C) Effective knockdown of HIF1A mRNA following lentivirus-mediated HIF1 $\alpha$ shRNA delivery into HepG2 cells (2-tailed, unpaired Student's $t$ test. $n=8$ and $n=7$ for the control [Ctrl] and shRNA groups, respectively). (D and E) HIF1 $\alpha$ shRNA abolished HIF1 $\alpha$ induction in HepG2 cell nuclei following 6 -hour hypoxia culturing in $1 \%$ oxygen (results are representative of 3 independent experiments). (G-I) Experiments analogous to those depicted in $\mathbf{D}$ and $\mathbf{E}$ demonstrate preserved induction of miR122 following shRNA-mediated repression of HIF2 $\alpha$. (G) Two-tailed, unpaired Student's $t$ test; $n=8$ and 11 for control and shRNA groups, respectively. ( $\mathbf{H}$ and $\mathbf{I}$ ) Results are representative of 4 independent experiments. ( $\mathbf{F}$ and $\mathbf{J}$ ) Time-dependent induction of miR122 in HepG2 cells exposed to hypoxia was abolished following HIF1 $\alpha$, but not HIF2 $\alpha$, knockdown compared with the same control shRNA group ( 2 -way ANOVA, $n=4$, and 6 at $0,2,4,8$ hours for the control group shared by $\mathbf{F}$ and $\mathbf{J}$, and $n=6$ for all shRNA groups, respectively, at $0,2,4$, and 8 hours). (K-N) In mice with hepatocyte-specific deletion of HIF1 $\alpha\left(H I F 1 A^{f / f f} A / b-C r e^{+}\right)$, HIF1A mRNA and protein were abolished, and induction of miR122 was abolished following ischemia and reperfusion (2-tailed, unpaired Student's $t$ test). (K) $n=5$ and 8 in WT and KO groups, respectively. (M) $n=3 / \mathrm{group}$. (N) $n=8$ and 6 in WT and KO groups, respectively. All data are shown as the mean \pm SEM. 


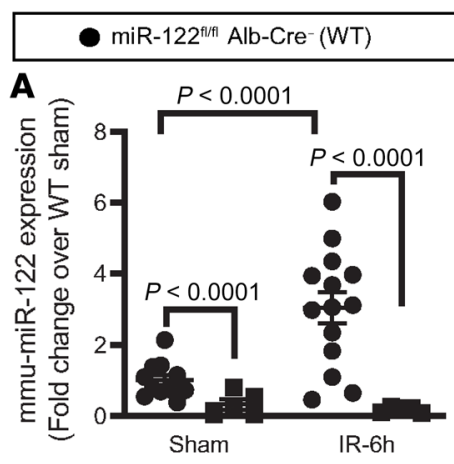

E
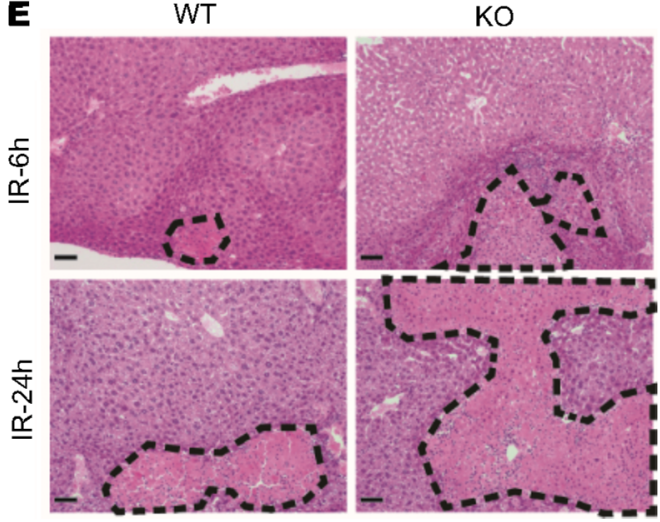

$\mathrm{miR}-122^{n / l f l} \mathrm{Alb}-\mathrm{Cre}+(\mathrm{KO})$

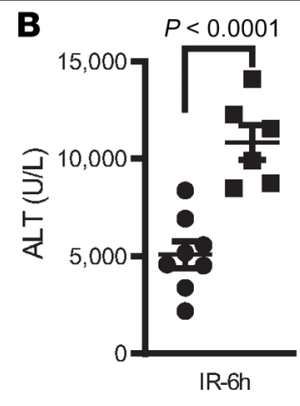

$\mathbf{F}$

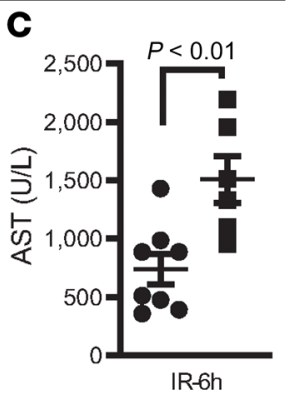

G

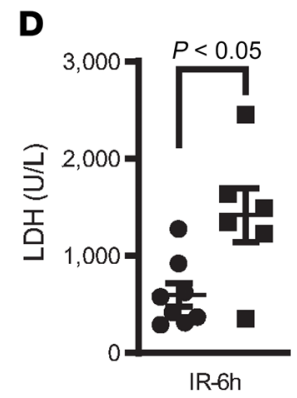

H
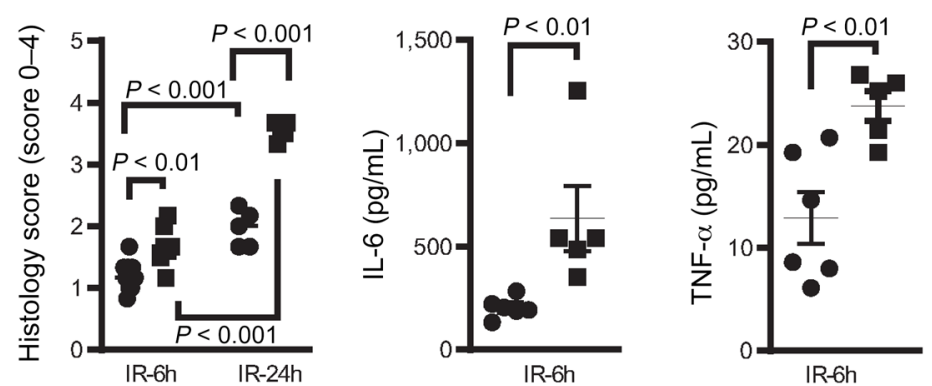

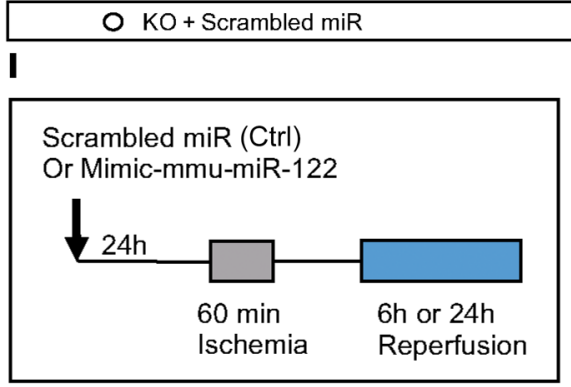

$\mathbf{N}$

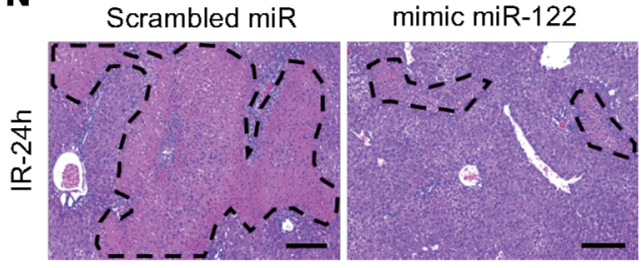

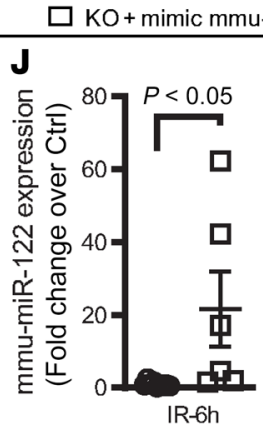

o

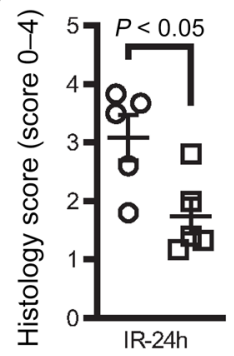

K

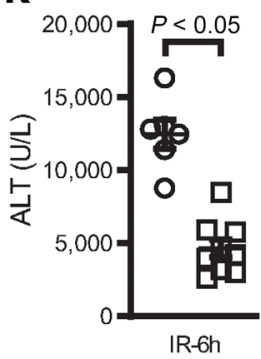

$\mathbf{P}$

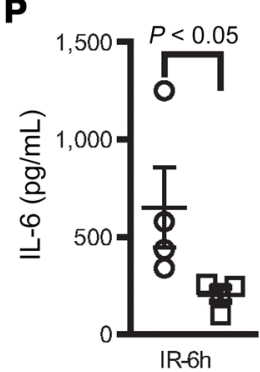

$\mathbf{L}$

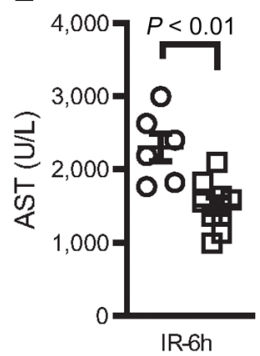

M

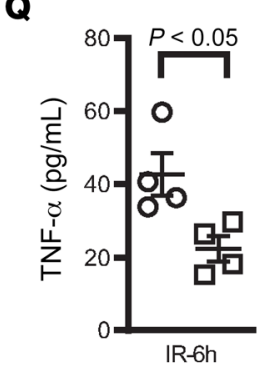

Figure 3. Exacerbated liver IR injury in mice with hepatocyte-specific deletion of miR122 is reduced by reconstitution of the miR122 mimic. Male miR122flff

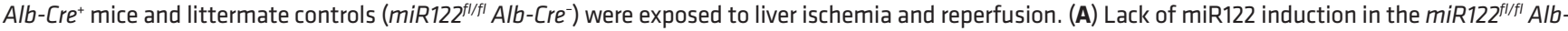
$\mathrm{Cr}^{+}$mice after 60 minutes of ischemia and 6 hours of reperfusion. Two-way ANOVA. $n=11,5$ in the sham WT and KO groups, respectively; $n=13,5$ in the IR WT and KO groups, respectively. miR122 fl/fl Alb-Cre+ mice showed increased hepatic injury, as determined by (B) ALT, (C) AST, and (D) LDH levels after 60 minutes of ischemia and 6 hours of reperfusion. Two-tailed Student's $t$ test. $n=8,6$ in the WT and KO groups, respectively. (E and F) Liver histology after 6 hours and 24 hours of reperfusion. Two-way ANOVA. $n=10,7$ for the 6-hour WT and KO groups, respectively; $n=5,7$ for the 24-hour WT and KO groups, respectively. Scale bars: $50 \mu \mathrm{m}$. (G and $\mathbf{H}$ ) Protein levels of the liver inflammation markers IL-6 and TNF- $\alpha$. Two-tailed, unpaired Student's $t$ test. $n=6$, 5 in the WT and KO groups, respectively. (I) miR122 fl/fl Alb-Cre+ mice were reconstituted with synthetic miRNA-122 or a scrambled miR control using nanoparticles 24 hours prior to 60 minutes of ischemia, followed by 6 or 24 hours of reperfusion. (J) Robust increases in miR122 levels were observed in the liver from mice reconstituted with a miR122 mimic compared with those reconstituted with a scrambled miR after ischemia and reperfusion. Two-tailed, unpaired Student's $t$ test. $n=9,6$ in the scrambled miR and miR122 groups, respectively. (K-M) Compared with miR122 fl/fl $\mathrm{Alb}$-Cre ${ }^{+}$mice that received scrambled miR treatment, mice reconstituted with synthetic miR122 show decreased levels of (K) ALT, (L) AST, and (M) LDH. $n=6$, 9 in the scrambled miR and miR122 groups, respectively. (N and $\mathbf{0}$ ) Histology images and improved histology scores. $n=5$ /group. Scale bars: $100 \mu \mathrm{m}$.(P and $\mathbf{Q})$ Reduced levels of the proinflammatory cytokines IL-6 and TNF- $\alpha$. $n=4$ /group. Two-tailed, unpaired Student's $t$ test. All data are shown as the mean \pm SEM. 
A Lentivirus
overexpression

B Lentivirus C overexpression
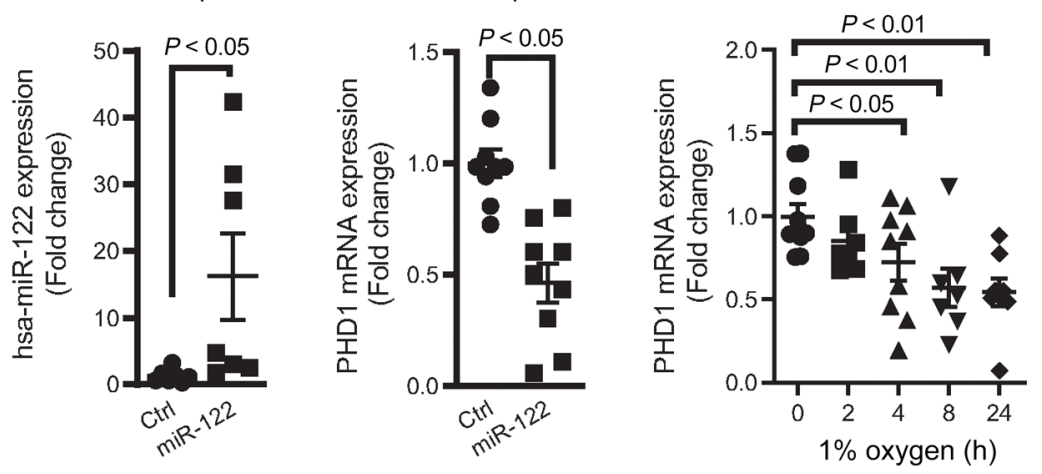

H
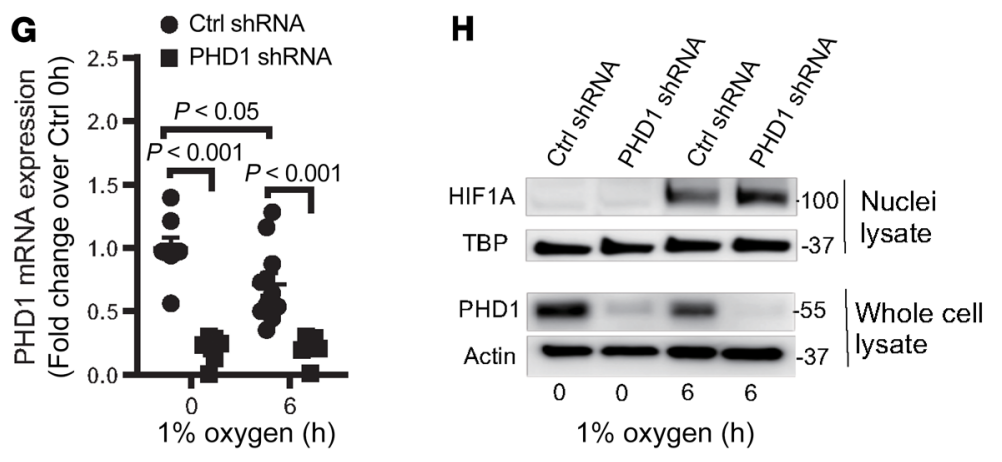

D PHD1 3'UTR -5' GGAGGCAUUGUCACUUCCCACCA 3' miR-122 -3' GUUUGUGUAACAGUGGAGGGAGGU $5^{\prime}$ PHD1 3'UTRmut -5' GGAGGUGCCAGGGUUUAACACCA 3'

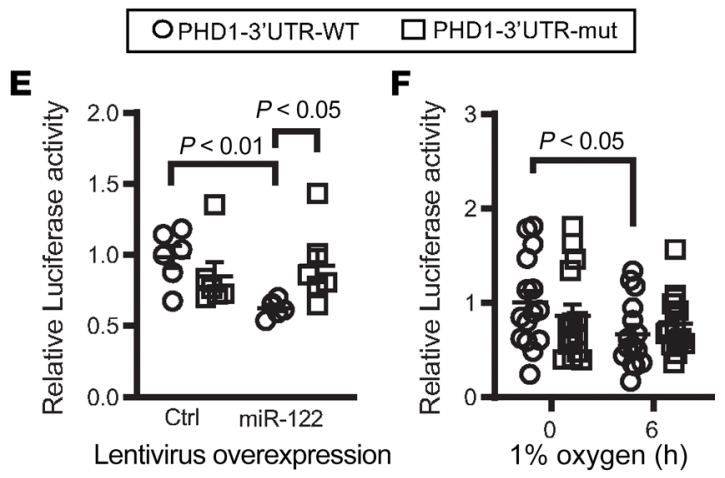

I Ctrl shRNA $\quad$ PHD1 shRNA

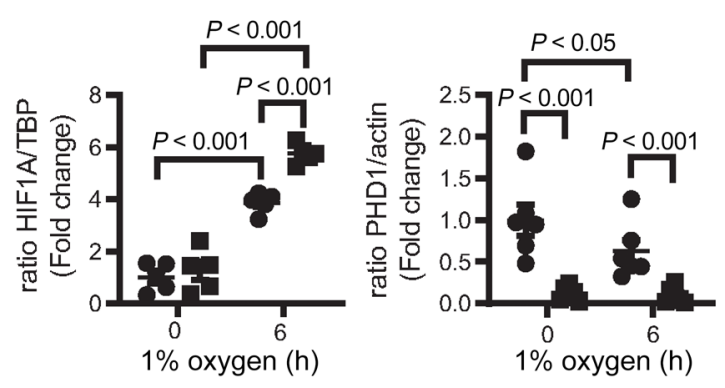

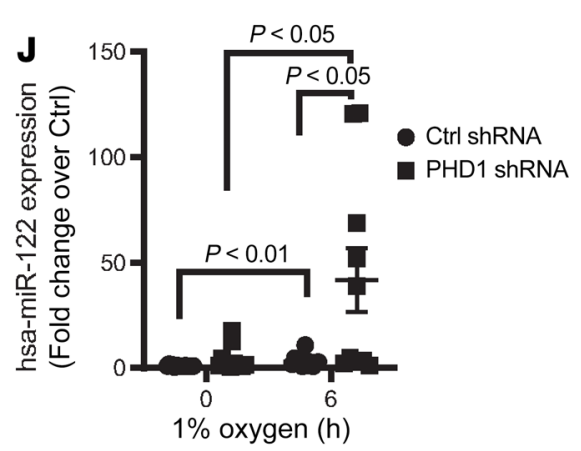
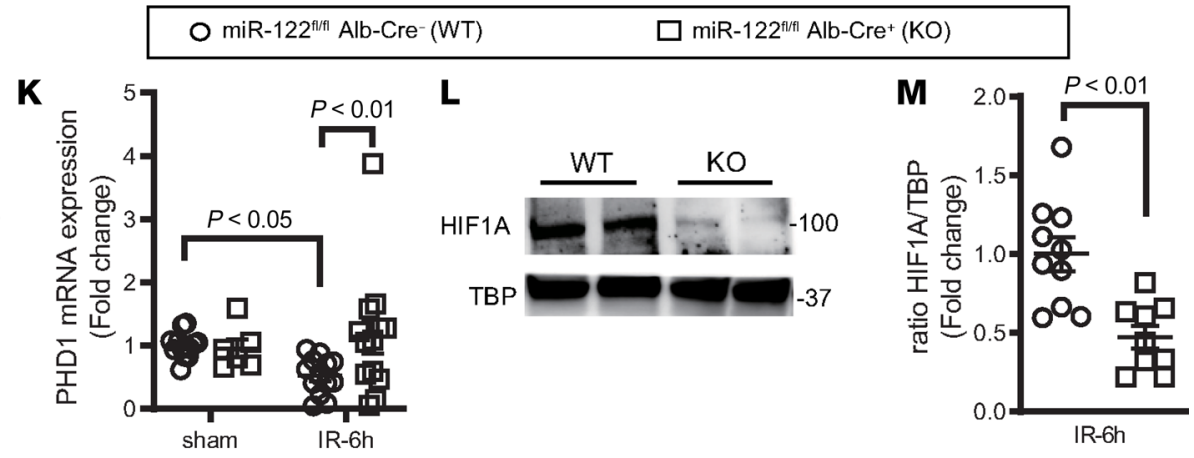

Figure 4. Identification of PHD1 as a miR122 target gene. (A and B) Hsa-miR122 levels and PHD1 transcript levels in HepG2 cells with miR122 overexpression compared with control cells. Two-tailed, unpaired Student's $t$ test. $n=7 /$ group (A); $n=9$ /group (B). (C) Repression of PHD1 transcripts in HepG2 cells following hypoxia exposure. One-way ANOVA. $n=10,7,9,7$, and 8 at $0,2,4,8$, and 24 hours, respectively. (D) Sequences used to generate 2 luciferase reporter plasmids, with 1 containing the WT 3'-UTR of PHD1, or a mutated version prohibiting binding of miR122 to the $3^{\prime}$-UTR. (E and F) Decreased luciferase activity caused by miR122 overexpression ( $n=6 /$ group) or following 24 hours of hypoxia ( $n=15 / g r o u p$ ) in HepG2 cells transfected with the WT PHD1-3'-UTR. These responses were absent in cells transfected with the mutated PHD1-3'-UTR plasmid (PHD1-3'-UTR-mut), where specific binding of miR122 to the 3'-UTR does not occur. A 2-way ANOVA was performed. (C) Effective PHD1 knockdown following lentivirus-mediated PHD1 shRNA delivery into HepG2 cells. $n=8,11$ in CTL-shRNA groups at 0 hours and 6 hours, respectively; $n=6$ in PHD1 shRNA groups at 0 hours and 6 hours. (H-J) HepG2 cells were cultured for 6 hours in hypoxic or normoxic conditions(0 hours). In PHD1-knockdown cells, hypoxia-induced HIF1 $\alpha$ protein stabilization ( $n=5 / g r o u p$ ) and miR122 augmentation were enhanced compared with control shRNA-transduced HepG2 cells. (J) $n=15,12$ in the control shRNA groups at 0 and 6 hours, respectively; $n=12,10$ in the PHD-1 shRNA groups at 0 and 6 hours, respectively. A 2-way ANOVA was performed. (K) PHD1 transcript expression was not suppressed in miR122 f/f Alb-Cre+ mice after 60 minutes of ischemia and 6 hours of reperfusion. Two-way ANOVA. $n=13,6$ in the sham WT and KO groups, respectively; $n=13$ /group in the IR groups. (L and $\mathbf{M}$ ) Lack of stabilization of HIF1 $\alpha$ in miR122 fl/fl Alb-Cre ${ }^{+}$mice compared with littermate controls. Two-tailed, unpaired Student's $t$ test. $n=10,9$ in the WT and KO groups, respectively. All data are shown as the mean \pm SEM.

inently regulated by a cluster of liver-specific transcription factors $(35,36)$. However, hypoxia control of the miR122 promoter is currently unknown. Notably, the human miR122 promoter (-1055 bp upstream of the transcription start site [TSS]) contains
2 putative hypoxia-inducing factor (HIF) binding sites (hypoxia response elements [HREs], -847 and -504; Figure 2A). ChIP assays demonstrated direct binding of HIF1 $\alpha$ to both HREs on the miR122 promoter (Figure 2B). Our data further demonstrated 


\section{Table 1. Screening of potential miR122 target genes}

\begin{tabular}{lccc} 
Gene name & Mean & SD & $\boldsymbol{P}$ value \\
\hline PHD1 (EGLN2) & 0.427 & 0.277 & $<0.001$ \\
\hline HNRNPU & 0.667 & 0.530 & 0.171 \\
GYS1 & 0.688 & 0.528 & 0.198 \\
\hline PHD1 (EGLN1) & 0.730 & 0.359 & 0.143 \\
\hline MAP3K12 & 0.931 & 0.611 & 0.570 \\
CCNG1 & 0.941 & 0.518 & 0.780 \\
\hline HIF3A & 1.015 & 1.337 & 0.985 \\
\hline HIF1AN & 1.101 & 0.509 & 0.743 \\
\hline DUSP4 & 1.1 .07 & 0.526 & 0.673 \\
VHL & 1.179 & 0.195 & 0.226 \\
\hline SLC2Z3 & 1.376 & 0.573 & 0.342 \\
RBPI & 1.463 & 0.822 & 0.418 \\
PKM & 1.464 & 0.409 & 0.108 \\
ATR & 1.489 & 1.182 & 0.390 \\
HIF1A & 1.659 & 0.637 & 0.022 \\
HMOX1 & 1.764 & 1.169 & 0.055 \\
ALDOA & 1.874 & 1.069 & 0.080 \\
RUVBL2 & 1.989 & 1.117 & 0.092 \\
P4HA1 & 2.008 & 0.640 & 0.023 \\
PHD3 (EGLN3) & 2.300 & 0.584 & 0.016 \\
EPO & 2.760 & 1.078 & $<0.001$
\end{tabular}

Putative miR122 target genes were measured in HepG2 cells after lentivirus-mediated miR122 overexpression. Representative results from 6 independent experiments are shown.

that hypoxia inducibility of miR122 was abolished in HepG2 cells with lentivirus-mediated shRNA repression of HIF1 $\alpha$ (Figure 2, $\mathrm{C}-\mathrm{F})$, whereas miR122 induction remained intact in HepG2 cells with shRNA-mediated repression of HIF $2 \alpha$ (Figure 2, G-J). Moreover, miR122 induction during hepatic IR injury was abolished in mice with hepatocyte-specific deletion of HIF1 $\alpha$ (HIF $1 A^{f l / f l}$ Alb$\mathrm{Cre}^{+}$mice; Figure 2, K-N). Together, these findings indicate that miR122 is a HIF1A target gene and implicate a functional role for HIF1 $\alpha$ in miR122 induction during hepatic IR injury.

Hepatocyte-specific deletion of miR122 (miR122 $2^{f / f l} \mathrm{Alb}-\mathrm{Cre}{ }^{+}$mice) is associated with exacerbated liver IR injury. Given the above studies showing HIF1 $\alpha$-dependent induction of miR122 during conditions of limited oxygen availability, we next sought to address the functional role of miR122 during hepatic IR injury. The previously described $\mathrm{miR} 122^{f / f l} \mathrm{Alb}$-Cre $\mathrm{C}^{+}$mice are viable, bred normally, and do not have obvious abnormalities when kept in a pathogen-free environment (19), but they show efficient deletion of hepatic miR122 levels (Figure 3A). However, exposure of $m i R 122^{f / f l} \mathrm{Alb}$ $\mathrm{Cr}^{+}$mice to hepatic IR injury revealed dramatically increased liver injury compared with their age-, sex-, and weight-matched littermates ( $m i R 122^{f l f l} \mathrm{Alb}-\mathrm{Cr} e^{-}$mice). This was evident from increases in ALT, AST, lactate dehydrogenase (LDH), histologic liver injury, and markers of liver inflammation (Figure 3, B-H). To further demonstrate that miR122 plays a functional role in the regulation

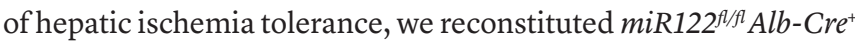
mice using treatment with nanoparticles containing a synthetic mouse miR122 mimetic via neutral lipid emulsion (NLE). The miR122 $2^{f / f l} \mathrm{Alb}-\mathrm{Cr} e^{+}$mice were intravenously injected with a single
$20 \mu \mathrm{g}$ dose of the miRNA-122 mimetic formulated with NLE or with an NLE containing a scrambled RNA sequence, 24 hours prior to hepatic ischemia surgery (Figure 3I). The exogenous miR122 treatment resulted in a significant increase in hepatic miR122 levels (Figure 3J), while functional studies demonstrated recapitulation of a WT phenotype with reduced levels of AST, ALT, LDH, histologic liver injury, and markers of liver inflammation (Figure $3, \mathrm{~K}-\mathrm{Q})$. Together, these studies provide strong evidence for a protective role of miR122 during hepatic IR injury.

Identification of prolyl hydroxylase domain 1 as a miR122 target gene. Given the profound increases in liver injury observed in $m i R$ $122^{f l / f l} \mathrm{Alb}$ - $\mathrm{Cre}^{+}$mice, we hypothesized that repression of a putative miR122 target gene could mediate hepatic ischemia tolerance. Using electronic miRNA target gene prediction software (PITA, RNAhybrid, miRecords, and TargetScanHuman), we identified several putative miRNA target genes with at least 2 positive predictions in the above search engines. To test these putative miR122 targets in a functional assay, we generated HepG2 cells with overexpression of hsa-miR122 (Figure 4A). A number of the genes reported to be miR122 targets showed a trending decrease following miR122 overexpression (Table 1). Interestingly, we observed the most profound and significant repression for the putative miR122 target gene prolyl hydroxylase domain 1 (PHD1) (Table 1 and Figure 4B). PHD1 is an oxygen-sensing prolyl hydroxylase that is critical for the regulation of HIF protein stabilization (2). Indeed, we found that hypoxia exposure of HepG2 cells was associated with the repression of PHD1 mRNA during ambient hypoxia (Figure 4C). We next generated 2 luciferase reporter plasmids with 1 containing the WT 3 '-UTR of PHD1 and a mutated version, in which specific binding of miR122 to the 3 '-UTR does not occur (Figure 4D). Transfection of these plasmids into HepG2 cells confirmed repression of the WT plasmid in the cells with miR122 overexpression or following hypoxia exposure - a response that was abolished following mutation of the PHD1-3'-UTR reporter plasmid (Figure 4, E and F). Together, these studies indicate that miR122 can function as a repressor of PHD1.

After having identified PHD1 as a hepatic miR122 target, we next performed studies to address the functional role of PHD1 repression. PHD1 is known to hydroxylate HIFs during normoxic conditions, thereby tagging HIF for proteasomal degradation (2). During limited oxygen availability, HIF hydroxylation is attenuated, leading to the initiation of hypoxia-elicited gene expression (2). Therefore, we hypothesized that miR122 repression of PHD1 could function to enhance hepatic HIF responses. To address this hypothesis, we generated HepG2 cells with lentivirus-mediated repression of PHD1 (Figure 4G). Indeed, hypoxia-induced stabilization of HIF1 $\alpha$ was significantly enhanced following PHD1 knockdown (Figure 4, H and I). Consistent with elevated HIF1 $\alpha$ signaling, we observed that miR122 expression was elevated in PHD1-knockdown HepG2 cells (Figure 4J). Moreover, we found that PHD1 transcript levels in mice with hepatocyte-specific miR122 deletion ( $m i R 122^{f / f l}$ Alb-Cre ${ }^{+}$mice) were not suppressed and that HIF1 $\alpha$ protein was not stabilized during hepatic IR injury (Figure 4, K-M). In the context of previous studies showing that $P h d 1^{-/-}$mice are protected during hepatic IR injury (37), the present findings indicate the likelihood that miR122-elicited repression of PHD1 could function to provide liver protection via enhancement of hepatic HIF responses. 


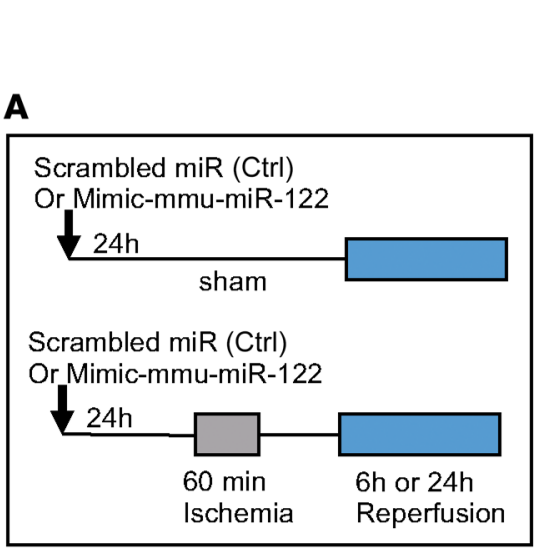

$\mathbf{F}$

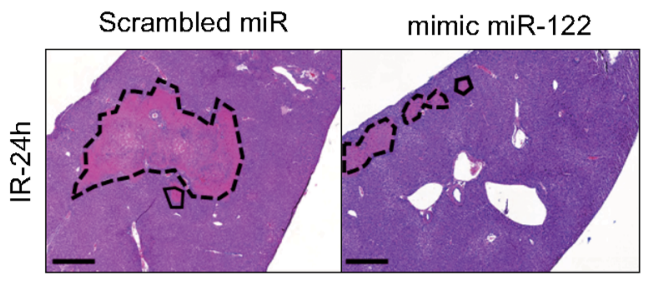

WT + Scrambled miR

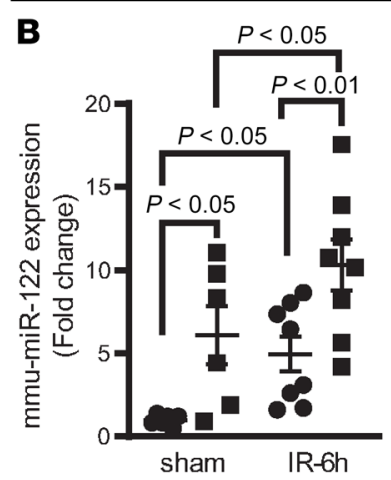

G

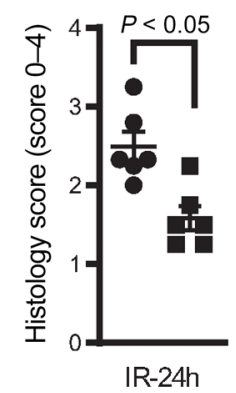

C

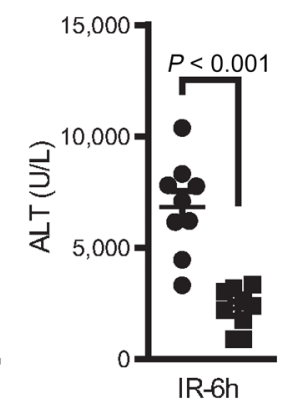

WT + mimic mmu-miR122

D

E
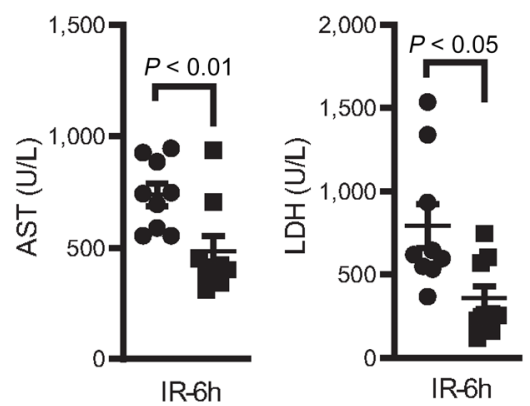

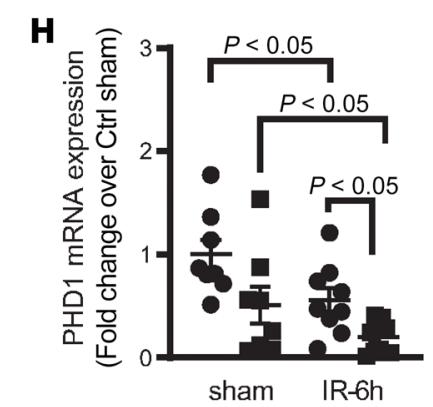

I

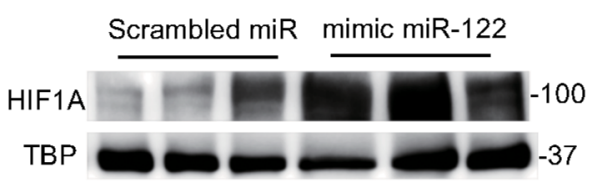

Nuclei lysate

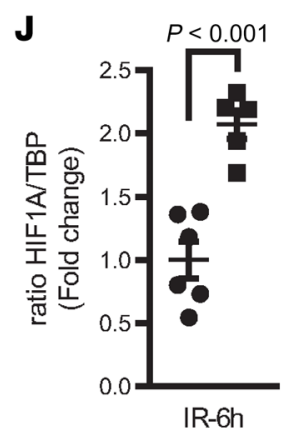

K

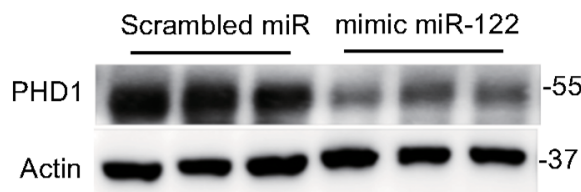

Whole tissue lysate
$\mathbf{L}$

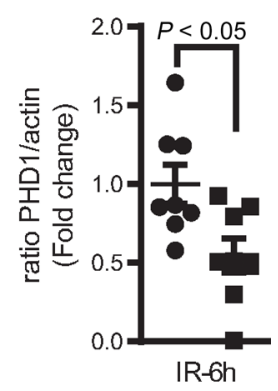

Figure 5. Hepatic overexpression of miR122 provides liver protection during hepatic IR injury. (A) Male C57BI/6 WT mice were injected intravenously with a single dose of $20 \mu \mathrm{g}$ synthetic mouse miR122 or scrambled miR formulated in NLE, 24 hours prior to 60 minutes of ischemia followed by 6 hours and 24

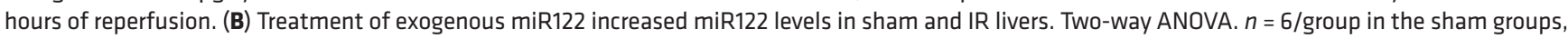
$n=8$ /group in the IR groups. Mice treated with the miR122 mimetic show decreased levels of ALT (C), AST (D), and LDH (E) following 6 hours of reperfusion. Two-tailed, unpaired Student's $t$ test. $n=9$ /group. ( $F$ and $\mathbf{G}$ ) Exogenous miR122 treatment improved histology scores following 24 hours of reperfusion.

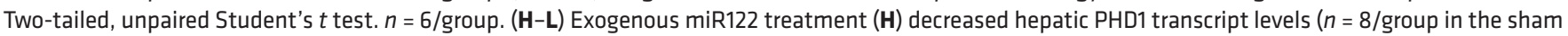
groups, $n=9$ /group in the IR groups), (I and J) augmented stabilization of HIF1 $(n=6,5$ in the scrambled miR and miR122 groups, respectively), and (K and L) repressed PHD1 protein levels ( $n=8 /$ group) after liver IR injury. Two-way ANOVA $(\mathbf{H})$ and 2-tailed, unpaired Student's $t$ test $(\mathbf{J}$ and $\mathbf{L})$.

Hepatic overexpression of miR122 provides liver protection during hepatic IR injury. To demonstrate the potential of pharmacologic interventions to directly enhance hepatic miR122 expression, we next performed studies of WT mice treated with nanoparticles containing a miR122 mimetic. Pretreatment with a single dose of the NLE-formulated miR122 mimetic 24 hours prior to ischemia surgery was associated with robust elevations of hepatic miR122 levels 6 hours after reperfusion (Figure 5, A and B). Studies of hepatic IR injury showed robust liver protection in the context of repressed PHD1 transcript and protein levels and elevated HIF1 $\alpha$ protein stabilization (Figure 5, C-L). Together, these studies indicate that pharmacologic induction of hepatic miR122 overexpression represents a therapeutic approach for the treatment of IR injury of the liver.
miR122 expression is increased in human hepatic IR injury. As a final step, we performed proof-of-principle studies in liver biopsies obtained during human liver transplantation. For this purpose, we obtained 2 liver biopsies from cadaveric liver transplantation the first during cold ischemia and a second biopsy sample from the same liver following warm ischemia and reperfusion. Each liver biopsy served as its own control ( $n=11$; average cold ischemia, warm ischemia, and reperfusion times are shown in Figure 6A; patients' characteristics are provided in Table 2). Liver biopsies were immediately snap-frozen in the operating room. Consistent with the above studies in murine IR injury of the liver, we found that hepatic hsa-miR122 levels were significantly elevated during human liver transplantation (Figure 6B). Consistent with a 
A

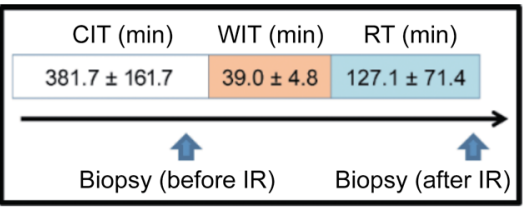

B

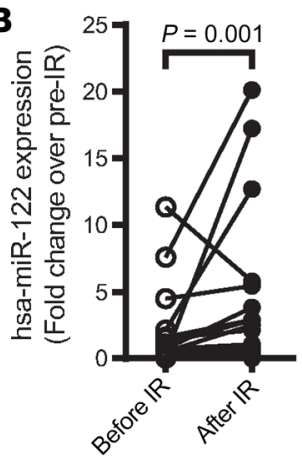

C

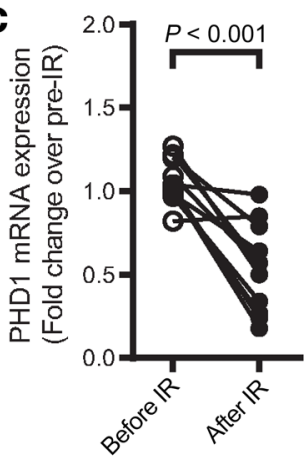

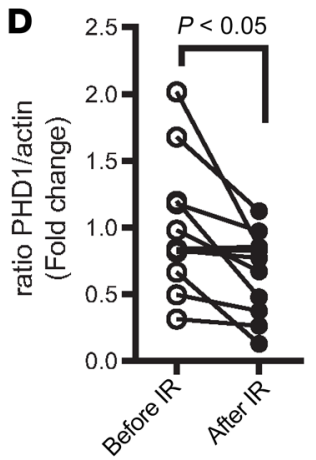

E

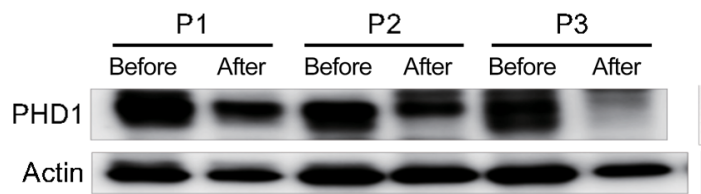

$\mathbf{F}$
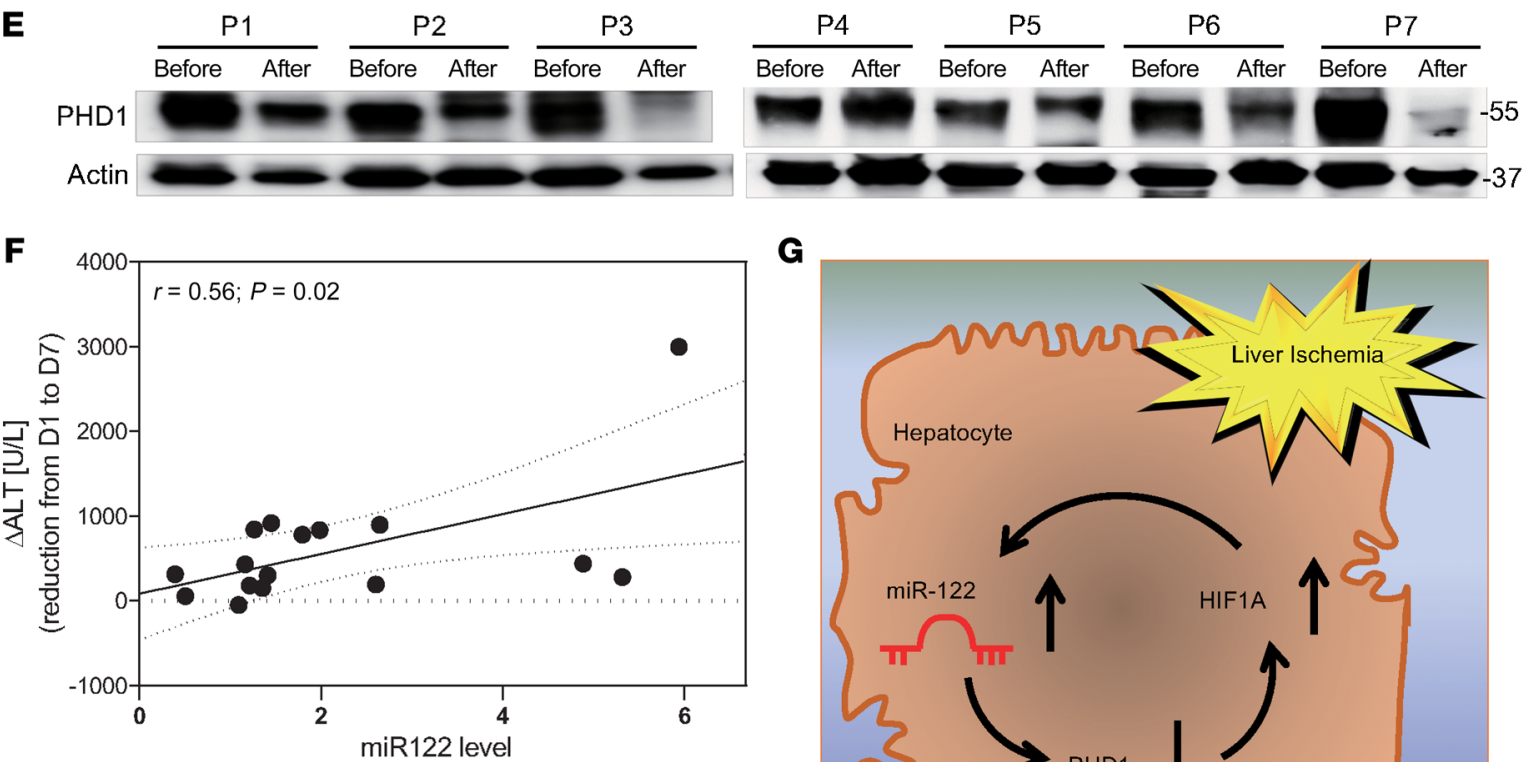

(Post-/pre-transplantation ratio)

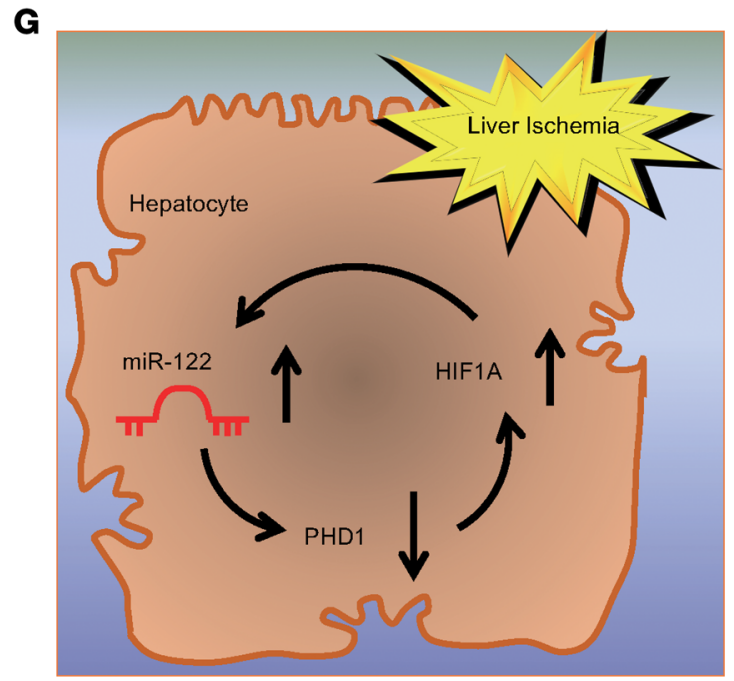

Figure 6. MiR122 expression is increased in human hepatic IR injury. (A) Two subsequent liver biopsies were obtained during cadaveric human liver transplantation - the first before IR and the second after warm ischemia and reperfusion after IR. Each liver biopsy served as its own control. (B and C) The miR122 ( $n=16$ /group) and PHD1 transcript levels ( $n=11$ /group) were measured by real-time RT-PCR. Two-tailed, paired Student's $t$ test. Data are shown as the mean \pm SEM. (D and E) PHD1 protein levels in pre- and post-IR liver samples were measured by Western blot analysis and quantified. Representative blots are shown. Two-tailed, paired Student's $t$ test. $n=11 /$ group. data are shown as the mean \pm SEM. (F) Correlation between the ratio of miR122 levels (post/pre-transplantation ratio) and the reduction in ALT levels from postoperative days 1 to 7 (D1 to D7) as an indicator of liver recovery ( $n=16$ samples). Data are depicted as linear regression (black line) with a 95\% Cl (dashed lines). (C) Schematic summary of the main findings. HIF1 $\alpha$-dependent upregulation of miR122 and miR122dependent repression of PHD1, which results in hepatic HIF1 $\alpha$ stabilization, represent a feed-forward pathway for liver protection during IR injury.

functional role for miR122 induction in repressing its target gene PHD1, we found that transcript and protein levels of PHD1 were repressed during human liver transplantation (Figure 6, C-E). Taken together, these findings indicate that the observed transcriptional alterations such as induction of miR122 and concomitant repression of PHD1 occur during human liver transplantation.

\section{Discussion}

The current studies are designed to identify miRNA targets for the treatment of hepatic IR injury. To make progress on this front, we performed a targeted miRNA screen in a murine model of partial liver ischemia. This approach revealed the most prominent increase in hepatic miR122 - a miRNA that has become famous for its role in propagating the replication of the hepatitis C virus (20). Interestingly, we observed a profound protective role for miR122 against hepatic IR injury, whereby mice with hepatocyte-specific deletion of miR122 experienced increased susceptibility to hepatic IR injury. In support of these findings, the ratio of miR122 levels in human patients (post-/pre-transplantation ratio) showed a significant correlation with the reduction in ALT levels from postoperative day 1 to day 7 as an indicator of liver recovery (Figure 6F). Subsequent studies demonstrated that miR122 is an HIF1 $\alpha$ target gene and that binding of HIF1 $\alpha$ to the miR122 promoter is critical for miR122 induction. Moreover, a subsequent study of hepatocytes with miR122 overexpression and further in vitro and in vivo experiments revealed that PHD1 


\section{Table 2. Patients' characteristics}

\begin{tabular}{lcccc} 
Repository no. & MELD score & CIT (min) & WIT (min) & BPT (min) \\
1 & 20 & 225 & 32 & 60 \\
2 & 28 & 723 & 36 & 80 \\
3 & 34 & 580 & 46 & 140 \\
4 & 40 & 271 & 36 & 124 \\
5 & 40 & 433 & 47 & 300 \\
6 & 28 & 571 & 45 & 115 \\
7 & 22 & 238 & 35 & 60 \\
8 & 33 & 538 & 40 & 196 \\
9 & 36 & 323 & 42 & 118 \\
10 & 40 & 381 & 38 & 71 \\
11 & 17 & 327 & 40 & 196 \\
12 & 31 & 178 & 38 & 78 \\
13 & 39 & 144 & 38 & 346 \\
14 & 30 & 432 & 39 & 233 \\
15 & 29 & 557 & 32 & 107 \\
16 & 38 & 314 & 42 & 145 \\
\hline Mean \pm SEM & $31.6 \pm 1.8$ & $389.7 \pm 41.7$ & $39.13 \pm 1.1$ & $148.1 \pm 21.4$
\end{tabular}

Individual patient and respective donor values are presented along with the mean $\pm S D$, including the model for end-stage liver disease (MELD) score, the cold ischemia time (CIT), the warm ischemia time (WIT), the reperfusion time (RT), and the time of biopsy after reperfusion (BPT).

is a target gene. As such, the present studies point toward a functional role of miR122 induction and concomitant repression of PHD1 as a feed-forward pathway to enhance hepatic HIF1 $\alpha$ stabilization and more robust HIF1a-elicited liver protection during hepatic IR injury (Figure 6G).

The HIF transcriptional complex, which is critical in cellular responses to hypoxia, was discovered in 1995 by Gregg Semenza (38), who was awarded the Nobel Prize in Physiology or Medicine in 2019. Several previous studies had implicated the PHD/ HIF pathway in promoting ischemia tolerance of different organs. For example, a recent study showed that loss of PHD1 lowers oxygen consumption in skeletal muscle by reprogramming glucose metabolism from oxidative to more anaerobic ATP production through activation of the PPAR $\alpha$ pathway (39). This metabolic adaptation to oxygen conservation impairs oxidative muscle performance in healthy conditions, but it provides acute protection of myofibers against lethal ischemia. In this model, hypoxia tolerance is due to reduced generation of oxidative stress, which allows PHD1-deficient myofibers to preserve mitochondrial respiration (39). Similarly, a recent study implicated an interaction of HIF1a with the circadian rhythm protein Period2 in promoting ischemia tolerance through the transcriptional induction of glycolytic enzymes, a pathway that can also be activated by light treatment (40). Moreover, inhibition of PHDs via succinate and concomitant stabilization of HIF1 $\alpha$ has been shown to optimize alveolar-epithelial carbohydrate metabolism, thereby attenuating lung inflammation during mechanical ventilation (41). Additional studies of ischemia and reperfusion demonstrated that HIF can function to promote the generation and signaling of antiinflammatory adenosine and thereby promote ischemia tolerance or dampen postischemic vasoconstriction and inflammation (42-44). Together, these studies indicate that PHD-dependent stabilization of HIF is a critical step in mediating ischemia tolerance $(45,46)$. In addition to miR122, our data showed substantial upregulation of miR217 upon hepatic ischemia and reperfusion. Emerging studies of mRNA splicing and miRNA target recognition suggest the importance of a "kissing complex" dimerization in the structure and folding stability of RNA-RNA interactions $(47,48)$. It is interesting to speculate that miR-217 may form a "kissing complex" with miR122 to promote tolerance to liver IR injury.

The present studies have important translational implications. As a direct extension of the present findings, therapeutic approaches to enhance hepatic miR122 via nanoparticle treatment with miR122 mimetics could be pursued in patients prior to liver transplantation. This could be achieved by treatment of the donor during cadaveric liver transplantation, the addition of miR122 mimetic-containing nanoparticles to the solution used for extracorporeal liver preservation, or by preoperative treatment of the donor prior to living-donor-related liver transplantation (49). In support of this notion, a recent study demonstrated that HIF1 $\alpha$ was responsive to cold hypoxia/reoxygenation (50). Because the liver functions as a filter for nanoparticles, overexpression of miRNAs in the liver is easier to achieve than in other organs (e.g., the heart). Alternatively, miR122-dependent liver protection can be mimicked by selective targeting of PHD1 using PHD1-specific oligonucleotides. Indeed, previous studies have shown that specific antisense oligonucleotides (ASOs) can be successfully used for the treatment of human disease (51-53). Alternatively, PHD inhibition could also be achieved pharmacologically by the use of PHD inhibitors. Indeed, a recent clinical study used a global PHD inhibitor successfully for the treatment of renal anemia in humans (54). As no side effects were reported, this study indicates safety for the use of PHD inhibitors in humans, and those findings could be extended toward human liver transplantation. However, a miRNA-based therapeutic approach could be more advantageous. For example, the inhibitory effect on PHD will last for a prolonged period (until the protein is recovered). This would be ideal for patients undergoing major liver surgery or liver transplantation, where postoperative oral intake is likely impossible for several days.

Finally, our findings also have important implications for patients with hepatocellular carcinoma (HCC). Emerging evidence suggests that miR122 is a tumor suppressor. miR122 expression is reduced in rodent and human HCC (55). Mice with genetic deletion of miR122, either globally or only in hepatocytes, spontaneously develop HCC after 1 year of life (56). Furthermore, delivery of exogenous miR122 suppressed liver tumor development in animal models (19). These studies suggest that miR122 supplementation during liver transplantation or resection will not only enhance tolerance against hepatic IR injury but might also prevent cancer recurrence in patients with HCC.

Taken together, the present studies implicate HIF1 $\alpha$-elicited induction of miR122 in hepatoprotection during IR injury via repression of its target gene PHD1. While these studies have important implications for liver transplantation or major liver surgery, steps to take these findings from bench to bedside will be critical. Such efforts could include testing pharmacologic approaches to achieve hepatic overexpression of miRNAs, or the design and safety testing of human ASOs for targeting of PHD1. 
Table 3. Primer sequences used in the studies

\begin{tabular}{|c|c|c|c|}
\hline Species & Gene & Forward primer $\left(5^{\prime}-3^{\prime}\right)$ & Reverse primer $\left(5^{\prime}-3^{\prime}\right)$ \\
\hline \multirow[t]{8}{*}{ Mouse } & $18 \mathrm{~s}$ & ACGGAAGGGCACCACCAGGA & САССАССАСССACGCAATCG \\
\hline & 116 & TCСATCCAGTTGССTTCTTG & TTCCACGATTTTCCCAGAGAAC \\
\hline & Tnfa & AGGCTGCCCCCGACTACGT & САСTTTCTCCTGGTATGAGATAGCAAA \\
\hline & Hifla & TTGCTCATCAGTTGCCACtT & TTAGCACCATCACAAAGCCA \\
\hline & Hif2a & CTGAGGAAGGAGAAATCCCGT & TGTGTCCGAAGGAAGCTGATC \\
\hline & $\operatorname{Phd1}(E g \ln 2)$ & QIAGEN (NM_053208, PPM60009B) & \\
\hline & Phd2(Egln1) & QIAGEN (NM_053207, PPM31485B) & \\
\hline & $\operatorname{Phd} 3(E g \ln 3)$ & QIAGEN (NM_028133, PPM31281A) & \\
\hline \multirow[t]{22}{*}{ Human } & $18 \mathrm{~s}$ & AACGAACGAGACTCTGGCAT & AАСGССАСТTGTСССТСТАA \\
\hline & ALDOA & CAGGGACAAATGGCGAGACTA & GGGGTGTGTTCCССАATCTT \\
\hline & $A T R$ & ACCTCAGCAGTAATAGTGATGGA & GGCCACTGTATTCAAGGGAAAT \\
\hline & DUPS4 & GGACСССАСТАCACGACCA & ATTCAACAGAGCCGTCATGCC \\
\hline & EPO & GTTTCGGCAAGTGAGGAGGA & GGCCCCAATCCAGATGTCAA \\
\hline & GYS1 & CAGCGCGGACCAACAATTTC & TCСTCССGAACTTTTCСTTCA \\
\hline & HIFIAN & TTCCCGACTAGGCCCATTC & CAGGTATTCAAGGTCCCATTTCA \\
\hline & HIF1A & ATCCATGTGACCATGAGGAAATG & TCGGCTAGTTAGGGTACACTTC \\
\hline & HIF2A & AGСАССССТСТСТСТTССАТ & GCTGCTCCCAAGAACTCTGT \\
\hline & HIF3A & ATGCGGTCAGCAAGAGCATC & AGACGATACTCTCCGACTGGG \\
\hline & HMOX1 & СCCACCAAGTTCAAACAGCT & ATСACCTGCAGCTCCTCAAA \\
\hline & HNRNPU & GAGCATCCTATGGTGTGTCAAA & TGACCAGCCAATACGAACTTC \\
\hline & МАРЗК12 & ССТCAAACAAACCGACATCAACC & GGATGCAGTAGCAGGGAGC \\
\hline & P4HA1 & ССAAAGCTCTGTTACGTCTCC & AGTCCTCAGCCGTTAGAAAAGA \\
\hline & PHD1 (EGLN2) & GCTGGGCAGCTATGTCATCAA & GGGATTGTCAACGTGCCTTAC \\
\hline & PHD2 (EGLN1) & GCTTGTTATCCGGGCAATGG & TGGGTTCAATGTCAGCAAACT \\
\hline & PHD3 (EGLN3) & CTGGGCAAATACTACGTCAAGG & GACCATCACCGTTGGGGTT \\
\hline & PKM & ATAACGCCTACATGGAAAAGTGT & TAAGCCCATCATCCACGTAGA \\
\hline & $R P B J$ & СGGCCTCCACCTAAACGAC & TCСATCСACTGCССATAAСAT \\
\hline & RUVBL2 & CATCCAGATTCATCGACCAGC & GAGCCCATAGCGTCGTAGT \\
\hline & SLC2A3 & GCTGGGCATCGTTGTTGGA & GCACTTTGTAGGATAGCAGGAAG \\
\hline & VHL & AGATGCAGGGACACACGATG & TTGACTAGGCTCCGGACAAC \\
\hline
\end{tabular}

\section{Methods}

Human liver tissue. Liver samples were obtained from patients undergoing orthotopic liver transplantation (Table 2). Liver biopsies (before IR) were taken at the conclusion of the cold ischemia time during back-table preparation of the cadaveric liver allograft (Figure 6A). A second biopsy (after IR) was taken immediately prior to closure of the abdomen following drain placement (Figure 6A). Importantly, total reperfusion time is defined as the time from portal vein perfusion to abdominal closure at the conclusion of the procedure.

Mice. Male mice were used for the studies. All animals were housed under a 12-hour light/12-hour dark cycle and were used (age- and weight-matched) between 12 and 16 weeks of age. $m i R 122^{f / f l}$ albumin $\mathrm{Cre}^{+}\left(\mathrm{miR} 122^{f / f l} \mathrm{Alb}-\mathrm{Cr} e^{+}\right)$mice were obtained by crossing $m i R 122^{f / / f l}$ mice with albumin $\mathrm{Cr}^{+}$mice. Littermate $\mathrm{miR} 122^{f / f l} \mathrm{Alb}$-Cre- mice were used as a control. $m i R 122^{f / f}$ mice were characterized previously by collaborators (19). HIF1 $A^{f l / f l} \mathrm{Alb}-\mathrm{Cr} e^{+}$mice were obtained by crossing $H I F 1 A^{f l f l}(40)$ and $A l b-C r e^{+}$mice. Alb-Cre mice were used as a control.
Murine model of partial liver ischemia. A murine model of partial liver ischemia was used as previously described (57). Mice were anesthetized by intraperitoneal injection of pentobarbital $(60 \mathrm{mg} /$ $\mathrm{kg}$ ). After a midline laparotomy, an atraumatic clip was used to block blood supply to the left lateral and median lobes of the liver. After 15-60 minutes of ischemia, the clip was be removed to initiate hepatic reperfusion.

Analysis of mRNA and miRNA levels. Total RNA was isolated from cultured cells or murine liver tissue using QIAzol Reagent and separated into mRNA and miRNA components following the manufacturer's instructions (SABiosciences, QIAGEN). cDNA from miRNA was generated using miScript RT II kits (QIAGEN), and transcript levels were determined by real-time reverse transcription PCR (RT-PCR) (iCycler; Bio-Rad). Primer sets for mmu-miR122 (MS00001526), hsa-miR122 (MS00003416), and RNU6 (MS00033740) were used following the manufacturer's instructions (QIAGEN). cDNA from mRNA was generated using iScript (Bio-Rad), and transcript levels were determined by real-time RT-PCR (iCycler; Bio-Rad). The primers sequences are shown in Table 3.

ChIP. ChIP was performed to assess DNA-protein interactions at the promoter sequences using the ChIP assay kit (Active Motif) according to the manufacturer's instructions. Briefly, cells were harvested, and protein-DNA complexes were crosslinked by fixation in $1 \%(\mathrm{v} / \mathrm{v})$ formaldehyde in minimal culture medium for 10 minutes at room temperature, followed by extraction of nuclei. Chromatin was enzymatically digested for 11 minutes to yield DNA fragments ranging in size from 200 to $1500 \mathrm{bp}$. The chromatin solution was reserved as the "input" sample. The remaining chromatin was immunoprecipitated overnight at $4^{\circ} \mathrm{C}$ with 3 $\mu \mathrm{g}$ antibody specific to HIF1 $\alpha$ (catalog 14179, Cell Signaling Technology) or an IgG isotype control (catalog 2729, Cell Signaling Technology). The chromatin-antibody complexes captured on the beads were washed several times and then eluted in $50 \mu \mathrm{L}$ elution buffer. The immunoprecipitated and input sample cross-links were reversed by incubation for 2.5 hours at $65^{\circ} \mathrm{C}$. After treatment with proteinase $\mathrm{K}$ for 1 hour at $37^{\circ} \mathrm{C}$, the reaction was stopped, and the resulting DNA was analyzed by realtime RT-PCR. The following primers were used: miR122 HRE-847 (sense, TGAAACCCCGTCTCTACTAA; antisense, ATTCAAGCGATTCTCCTGCCTC) and miR122 HRE-504 (sense, GACTGTGGGCTCTGTTTGT; antisense, ACCATGCATTCCTTTGTT).

In vitro transduction of virus overexpressing GFP-miR122. HepG2 cells (American Type Culture Collection [ATCC]) were transduced with $3 \times 10^{6}$ infectious units (IFU) of either GFP control- or GFP hsamiR122-expressing virus in $8 \mu \mathrm{g} / \mathrm{mL}$ polybrene (MilliporeSigma). After 48 hours, cells were harvested and mRNA was isolated using the RNeasy kit (QIAGEN). cDNA was generated using iScript cDNA Synthesis kits (Bio-Rad). 
Binding of miR122 to the $3^{\prime}$-UTR of PHD1. For overexpression, HepG2 cells were transduced with $3 \times 10^{6}$ IFU of either GFP control or GFPmiR122 virus and $8 \mu \mathrm{g} / \mathrm{mL}$ polybrene (MilliporeSigma). After 48 hours, cells were transfected with the control pMiR target or with PHD1-3'-UTR reporter constructs (OriGene). Twenty-four hours later, cells were harvested, and luciferase expression was measured using the Dual Luciferase Reporter Kit (Promega) according to the manufacturer's instructions. For hypoxia, HepG2 cells were transfected with a control pMirTarget or PHD1-3'-UTR reporter constructs (OriGene), incubated for 24 hours, and then exposed to $1 \%$ oxygen for 6 hours. Next, cells were harvested, and luciferase expression was measured using the Dual Luciferase Reporter kit (Promega) according to the manufacturer's instructions.

Immunoblotting experiments. To measure PHD1, HIF $1 \alpha$, or HIF $2 \alpha$ protein content, nuclear and cytoplasmic proteins were extracted from cells or liver tissue using NE-PER Nuclear and Cytoplasmic Extraction Reagents Complete Protease Inhibitor (Cell Signaling Technology). The protein was resuspended in reducing Laemmli sample buffer and heated to $95^{\circ} \mathrm{C}$ for 5 minutes. Samples were resolved on a $7.5 \%$ polyacrylamide gel and transferred onto nitrocellulose membranes. The membranes were blocked for 1 hour at room temperature in TBS Tween-20 (TBST) supplemented with 5\% BSA (MilliporeSigma). The membranes were incubated overnight at $4{ }^{\circ} \mathrm{C}$ with the following primary antibodies, at the indicated concentrations: rabbit anti-mouse PHD1 or anti-human PHD1 (catalog ab113077, Abcam, 1:1000); mouse anti-human HIF1 $\alpha$ (catalog 610959, BD, 1:500); and rabbit anti-mouse HIF1 $\alpha$ (catalog ab179483, Abcam, 1:1000) or HIF2 $\alpha$ (cata$\log$ NB100-122, Novus, 1:50). After three 10-minute washes in TBST, the membranes were incubated with a goat anti-mouse (catalog 7076, Cell Signaling Technology) or goat anti-rabbit (catalog 7074, Cell Signaling Technology) secondary antibody at a concentration of 1:2000. The wash was repeated and proteins detected by ECL (Pierce, Thermo Fisher Scientific). To control for nuclear protein loading, blots were probed for TATA-binding protein (TBP) (catalog 44059S, Cell Signaling Technology), and to control for cytosol protein loading, blots were probed for $\beta$-actin (A5441, MilliporeSigma).

PHD1 genetic deletion in cell cultures. HepG2 cells were transfected with pLKO.1-puro-PHD1 KO (PHD1-KO, $1 \mu \mathrm{g}$ ) or pLKO.1-puro $(1 \mu \mathrm{g})$ using FuGENE HD Transfection Reagent (Promega). Cells transfected with the pLKO.1-puro plasmid alone, without the PHD1-KO gene, were used as a control. For stable transfection, cells were cultured in the selective medium with $2 \mu \mathrm{g} / \mathrm{mL}$ puromycin for 1 month. Then, drug-resistant individual clones were isolated and incubated for further amplification in the presence of a selective medium.

In vivo treatment with miR122 mimic. NLE (maxSuppressor in vivo RNALancer II) was purchased from Bioo Scientific. NLE consists of 1,2-dioleoyl-sn-glycero-3-phosphocholine, squalene oil, polysorbate 20 , and an antioxidant that, in complex with synthetic miRNAs, forms nanoparticles in the nanometer-diameter range. A single dose of $20 \mu \mathrm{g}$ synthetic mouse microRNA-122 (Dharmacon), formulated with NLE according to the manufacturer's instructions, was injected intravenously into mice 24 hours before liver IR surgery.
Statistics. Liver injury score data are given as the median and the range. All other data are presented as the mean \pm SEM for $3-13$ animals per condition. Statistical analysis was performed using the Student's $t$ test for 2 experimental groups evaluated by the 2-sample equal variance $t$ test; a 1-way ANOVA for multiple experimental groups involving 1 factor; or a 2-way ANOVA for experiments involving 2 or 3 factors. A $P$ value of less than 0.05 was considered statistically significant. For Western blot analyses, experiments were repeated 3 times. GraphPad Prism 7.0 (GraphPad Software) was used for all statistical analyses.

Study approval. The collection and use of patients' samples was approved by the Committee for the Protection of Human Subjects (CPHS) at the University of Texas Health Science Center (UTHealth, CPHS protocol HSC-MS-12-0652). All patients included in the study provided written informed consent. All animal protocols were conducted in accordance with UTHealth guidelines for the use of living animals and were approved by the Center for Laboratory Animal Medicine and Care (CLAMC) at UTHealth.

\section{Author contributions}

CJ conceptualized the study, designed the experiments, interpreted the data, and wrote the manuscript and was thus assigned the first co-first authorship position. MW conducted the majority of the experiments, analyzed the data, and prepared the figures and was thus assigned the second co-first authorship position. ET conducted less than half of the experiments and analyzed the data and was thus assigned the third co-first authorship position. XY and BK conducted some of the experiments, analyzed the data, and reviewed the manuscript. YL, WAD, DRH, and JSB provided patients' samples, analyzed clinical data, and reviewed the manuscript. PC provided technical and conceptual support for studies of PHD1/HIF1 $\alpha$ signaling and reviewed the manuscript. YY, CE, and PC analyzed the data and reviewed the manuscript. HK and KG provided the hepatocyte-specific miR122-KO mice and technical support and reviewed the manuscript. HKE conceived and supervised the project and wrote the manuscript.

\section{Acknowledgments}

We wish to thank Sean Colgan (University of Colorado Anschutz Medical Campus) for his valuable advice and critique of the manuscript. The current studies were supported by NIH grants R01DK109574, R01DK121330, and R01DK122708 (to CJ) and by the Ertan Research and Educational Fund (to CJ). This work was also supported by NIH grants R01DK088076-05A1 (to KG), R01 DK097075, R01-HL0921, R01-DK083385, R01-HL098294, and POIHL114457-01 (to HKE), and by the Crohn's and Colitis Foundation of America (CCFA) (to HKE).

Address correspondence to: Cynthia Ju, 6431 Fannin Street, MSB 6.246; Houston, Texas 77030, USA. Phone: 713.500.7424; Email: Changqing.Ju@uth.tmc.edu.
1. Eltzschig HK, Eckle T. Ischemia and reperfusion--from mechanism to translation. Nat Med. 2011;17(11):1391-1401

2. Eltzschig HK, Carmeliet P. Hypoxia and inflammation. NEngl JMed. 2011;364(7):656-665.
3. Hilmi I, et al. The impact of postreperfusion syndrome on short-term patient and liver allograft outcome in patients undergoing orthotopic liver transplantation. Liver Transpl. 2008;14(4):504-508.
4. Cameron AM, et al. Optimal utilization of donor grafts with extended criteria: a single-center experience in over 1000 liver transplants. Ann Surg. 2006;243(6):748-753.

5. Pirenne J, et al. Influence of ischemia-reperfusion 
injury on rejection after liver transplantation. Transplant Proc. 1997;29(1-2):366-367.

6. Watt KD, et al. Recurrent hepatitis $C$ posttransplant: early preservation injury may predict poor outcome. Liver Transpl. 2006;12(1):134-139.

7. Kageyama S, et al. Recombinant relaxin protects liver transplants from ischemia damage by hepatocyte glucocorticoid receptor: From benchto-bedside. Hepatology. 2018;68(1):258-273.

8. Park SW, et al. Paneth cell-derived IL-17A causes multi-organ dysfunction after hepatic ischemia and reperfusion injury. Hepatology. 2011;53(5):1662-1675.

9. Liu Y, et al. Recipient T cell TIM-3 and hepatocyte galectin-9 signalling protects mouse liver transplants against ischemia-reperfusion injury. $J$ Hepatol. 2015;62(3):563-572.

10. Ke B, et al. KEAP1-NRF2 complex in ischemia-induced hepatocellular damage of mouse liver transplants. J Hepatol. 2013;59(6):1200-1207.

11. Li C, et al. Hippo signaling controls NLR family pyrin domain containing 3 activation and governs immunoregulation of mesenchymal stem cells in mouse liver injury. Hepatology. 2019;70(5):1714-1731.

12. Liu Y, et al. Activation of YAP attenuates hepatic damage and fibrosis in liver ischemia-reperfusion injury. J Hepatol. 2019;71(4):719-730.

13. Nakamura K, et al. Antibiotic pretreatment alleviates liver transplant damage in mice and humans. JClin Invest. 2019;129(8):3420-3434.

14. Burnstock $\mathrm{G}$, et al. Purinergic signalling in the liver in health and disease. Purinergic Signal. 2014;10(1):51-70.

15. Lee TJ, et al. Strategies to modulate MicroRNA functions for the treatment of cancer or organ injury. Pharmacol Rev. 2020;72(3):639-667.

16. Lagos-Quintana $M$, et al. Identification of tissue-specific microRNAs from mouse. Curr Biol. 2002;12(9):735-739.

17. Krutzfeldt J, et al. Silencing of microRNAs in vivo with 'antagomirs'. Nature. 2005;438(7068):685-689.

18. Esau C, et al. miR122 regulation of lipid metabolism revealed by in vivo antisense targeting. Cell Metab. 2006;3(2):87-98.

19. Hsu SH, et al. Essential metabolic, anti-inflammatory, and anti-tumorigenic functions of miR122 in liver.JClin Invest. 2012;122(8):2871-2883.

20. Janssen HL, et al. Treatment of HCV infection by targeting MicroRNA. N Engl JMed. 2013;368(18):1685-1694.

21. Satishchandran A, et al. MicroRNA 122, Regulated by GRLH2, protects livers of mice and patients from ethanol-induced liver disease. Gastroenterology. 2018;154(1):238-252.

22. Russo MW, et al. Profiles of miRNAs in serum in severe acute drug induced liver injury and their prognostic significance. Liver Int. 2017;37(5):757-764.

23. Chowdhary V, et al. miRNA-122 protects mice and human hepatocytes from acetaminophen toxicity by regulating cytochrome $\mathrm{P} 450$ family 1 subfamily A member 2 and family 2 subfamily E member 1 expression. Am J Pathol. 2017;187(12):2758-2774.

24. Bala $S$, et al. Circulating microRNAs in exosomes indicate hepatocyte injury and inflammation in alcoholic, drug-induced, and inflammatory liver diseases. Hepatology. 2012;56(5):1946-1957.

25. Bihrer V, et al. Serum miR122 as a biomarker of necroinflammation in patients with chronic hepatitis $\mathrm{C}$ virus infection. Am J Gastroenterol. 2011;106(9):1663-1669.

26. Trebicka J, et al. Hepatic and serum levels of miR122 after chronic HCV-induced fibrosis. JHepatol. 2013;58(2):234-239.

27. Pirola CJ, et al. Circulating microRNA signature in non-alcoholic fatty liver disease: from serum non-coding RNAs to liver histology and disease pathogenesis. Gut. 2015;64(5):800-812.

28. Roderburg C, et al. Elevated miR122 serum levels are an independent marker of liver injury in inflammatory diseases. Liver Int . 2015;35(4):1172-1184

29. van Caster $\mathrm{P}$, et al. Circulating microRNA-122, -21 and -223 as potential markers of liver injury following warm ischaemia and reperfusion in rats. Mol Med Rep. 2015;12(2):3146-3150.

30. Selten JW, et al. The release of microRNA-122 during liver preservation is associated with early allograft dysfunction and graft survival after transplantation. Liver Transpl.2017;23(7):946-956.

31. Wang K, et al. Circulating microRNAs, potential biomarkers for drug-induced liver injury. Proc Natl Acad Sci U S A. 2009;106(11):4402-4407.

32. Wang B, et al. Role of microRNA-155 at early stages of hepatocarcinogenesis induced by choline-deficient and amino acid-defined diet in C57BL/6 mice. Hepatology. 2009;50(4):1152-1161.

33. Dolganiuc A, et al. MicroRNA expression profile in Lieber-DeCarli diet-induced alcoholic and methionine choline deficient diet-induced nonalcoholic steatohepatitis models in mice. Alcohol Clin Exp Res. 2009;33(10):1704-1710.

34. Tang Y, et al. Effect of alcohol on miR-212 expression in intestinal epithelial cells and its potential role in alcoholic liver disease. Alcohol Clin Exp Res. 2008;32(2):355-364.

35. Li ZY, Xi Y, Zhu WN, Zeng C, Zhang ZQ, Guo ZC, et al. Positive regulation of hepatic miR122 expression by HNF4a.J Hepatol. 2011;55(3):602-611.

36. $\mathrm{Xu} \mathrm{H}$, et al. Liver-enriched transcription factors regulate microRNA-122 that targets CUTL1 during liver development. Hepatology. 2010;52(4):1431-1442.

37. Schneider M, et al. Loss or silencing of the PHD1 prolyl hydroxylase protects livers of mice against ischemia/reperfusion injury. Gastroenterology. 2010;138(3):1143-1154.

38. Wang GL, Semenza GL. Purification and characterization of hypoxia-inducible factor 1. J Biol Chem. 1995;270(3):1230-1237.

39. Aragones J, et al. Deficiency or inhibition of oxygen sensor Phd1 induces hypoxia tolerance by reprogramming basal metabolism. Nat Genet.
2008;40(2):170-180.

40. Eckle T, et al. Adora2b-elicited Per2 stabilization promotes a HIF-dependent metabolic switch crucial for myocardial adaptation to ischemia. Nat Med. 2012;18(5):774-782.

41. Eckle T, et al. HIF1A reduces acute lung injury by optimizing carbohydrate metabolism in the alveolar epithelium. PLoS Biol. 2013;11(9):e1001665.

42. Sitkovsky MV, et al. Physiological control of immune response and inflammatory tissue damage by hypoxia-inducible factors and adenosine $\mathrm{A} 2 \mathrm{~A}$ receptors. Annu Rev Immunol. 2004;22(1):657-682.

43. Eckle T, et al. Identification of hypoxia-inducible factor HIF-1A as transcriptional regulator of the A2B adenosine receptor during acute lung injury. JImmunol. 2014;192(3):1249-1256.

44. Sitkovsky M, Lukashev D. Regulation of immune cells by local-tissue oxygen tension: HIF1 $\alpha$ and adenosine receptors. Nat Rev Immunol. 2005;5(9):712-721.

45. Aragones J, et al. Oxygen sensors at the crossroad of metabolism. Cell Metab. 2009;9(1):11-22.

46. Fraisl P, et al. Inhibition of oxygen sensors as a therapeutic strategy for ischaemic and inflammatory disease. Nat Rev Drug Discov. 2009;8(2):139-152.

47. Cao S, Chen SJ. Structure and stability of RNA/RNA kissing complex: with application to HIV dimerization initiation signal. $R N A$. 2011;17(12):2130-2143.

48. Windbichler N, et al. Kissing complex-mediated dimerisation of HIV-1 RNA: coupling extended duplex formation to ribozyme cleavage. Nucleic Acids Res. 2003;31(22):6419-6427.

49. Trotter JF, et al. Adult-to-adult transplantation of the right hepatic lobe from a living donor. $N \mathrm{Engl}$ JMed. 2002;346(14):1074-1082.

50. Dery KJ, et al. Human antigen R (HuR): a new regulator of heme oxygenase- 1 cytoprotection in mouse and human liver transplant injury. Hepatology. 2020;72(3):1056-1072.

51. Kastelein JJ, et al. Potent reduction of apolipoprotein B and low-density lipoprotein cholesterol by short-term administration of an antisense inhibitor of apolipoprotein B. Circulation. 2006;114(16):1729-1735.

52. Bennett CF, et al. Pharmacology of antisense drugs. Annu Rev Pharmacol Toxicol. 2017;57:81-105.

53. Crooke ST, et al. RNA-targeted therapeutics. Cell Metab. 2018;27(4):714-739.

54. Bernhardt WM, et al. Inhibition of prolyl hydroxylases increases erythropoietin production in ESRD. J Am Soc Nephrol. 2010;21(12):2151-2156.

55. Kutay H, et al. Downregulation of miR122 in the rodent and human hepatocellular carcinomas. JCell Biochem. 2006;99(3):671-678

56. Tsai WC, et al. MicroRNA-122 plays a critical role in liver homeostasis and hepatocarcinogenesis. JClin Invest. 2012;122(8):2884-2897.

57. Abe Y, et al. Mouse model of liver ischemia and reperfusion injury: method for studying reactive oxygen and nitrogen metabolites in vivo. Free Radic Biol Med. 2009;46(1):1-7. 